\title{
Culture models to study leukocyte trafficking across the choroid plexus
}

\author{
Tobias Tenenbaum ${ }^{*}$, Ulrike Steinmann ${ }^{1}$, Corinna Friedrich ${ }^{2}$, Jürgen Berger ${ }^{3}$, Christian Schwerk $^{1}$ \\ and Horst Schroten ${ }^{1}$
}

\begin{abstract}
Background: A critical point during the course of central nervous system infection is the influx of leukocytes from the blood into the brain across the blood-brain barrier (BBB) and the blood-cerebrospinal fluid barrier (BCSFB). However, experimental in vitro models to investigate leukocyte transmigration across cultured choroid plexus epithelial cells have been lacking so far.

Methods: We have developed a porcine and human "inverted" culture insert system that enables leukocyte transmigration specifically from the physiologically relevant basolateral side. The models use primary porcine choroid plexus epithelial cells (PCPEC) and human choroid plexus papilloma cells (HIBCPP). As a prerequisite for a functional barrier, we optimized culture conditions in which cells are maintained in serum-containing medium until high barrier function is reached. Leukocyte transmigration through the plexus epithelial cells is analysed by three-dimensional Apotome ${ }^{\circledR}$-imaging and electron microscopy, and the route of transmigration through the plexus epithelial cells, i.e. transcellular as well as paracellular, can be determined.

Discussion: As a functionally relevant porcine and human BCSFB model, PCPEC and HIBCPP respectively, offer a wide range of options for analysis of disease-related mechanisms at the choroid plexus epithelium, especially involving human pathogens. Moreover, our in vitro models facilitate the investigation of leukocyte entry into the CNS via the blood-CSF barrier.
\end{abstract}

Keywords: Blood-cerebrospinal fluid barrier, Leukocyte, Transmigration, Meningitis

\section{Background}

The central nervous system (CNS) is separated from the blood by specific cellular structures including the bloodbrain barrier $(\mathrm{BBB})$ and the blood-cerebrospinal fluid (CSF) barrier (BCSFB). Whereas the endothelial cells of the brain microvasculature form the $\mathrm{BBB}$, the structural basis for the blood-CSF barrier is the choroid plexus epithelium. The epithelial cells of the choroid plexus are closely connected to each other by a belt of tight junctions (TJs). TJs of epithelial and endothelial cells maintain the asymmetry of the plasma membrane and serve as a regulated permeability barrier for paracellular transepithelial and transendothelial transport of

\footnotetext{
* Correspondence: Tobias.Tenenbaum@umm.de

${ }^{1}$ Paediatric Infectious Diseases, Department of Pediatric and Adolescent Medicine, Universitätsmedizin Mannheim, Medical Faculty Mannheim, Heidelberg University, Theodor-Kutzer-Ufer 1-3, Mannheim, Germany Full list of author information is available at the end of the article
}

physiologically important solutes, the penetration of microorganisms and other antigenic material [1]. Other important functions of the choroid plexus are maintenence of homeostasis in the CNS and CSF-secretion and participation in neurohumoral brain modulation and neuroimmune interactions [2,3]. In the case of the endothelium at the $\mathrm{BBB}$, these cells are interconnected by a dense network of TJs and they exhibit a low pinocytotic activity concomitant with the absence of fenestrae [4]. Properties of these cellular barriers are a high transendothelial or transepithelial electrical resistance (TEER) as well as a low permeability for macromolecules [5].

There is increasing evidence that the choroid plexus plays an important role in CNS inflammation, but the exact role of the BCSFB in this context is still under investigation [6,7]. The most comprehensively-investigated model for CNS inflammation is experimental autoimmune encephalomyelitis (EAE), which is considered

\section{Biomed Central}


the prototype model for the human disease multiple sclerosis (MS) [3]. But also bacterial and viral meningitis are important causes of mortality and morbidity despite advances in antimicrobial therapy. For many important meningitis pathogens such as Neisseria meningitides $(N$. meningitides) [8], Haemophilis influenzae (H. influenzae) [9], Escherichia coli (E. coli) [10,11], Listeria monocytogenes (L. monocytogenes) [11], Streptococcus suis (S. suis) [12] and enteroviruses [13], experimental data suggest involvement of the choroid plexus during bacterial entry into the brain. Most cases of meningitis develop as a result of haematogenous spread, but it is unclear how circulating bacteria cross the blood-brain barriers. Moreover, the mechanisms by which pathogens enter the CNS, lead to inflammation, pleocytosis (with predominantly polymorphonuclear neutrophils (PMN) in bacterial and lymphocytes/monocytes in viral meningitis) blood-brain barrier disruption and neuronal injury are still under investigation $[14,15]$.

During inflammatory events the BBB and BCSFB undergo major alterations, which lead to an opening of TJs, break-down of barrier function and massive influx of immune system cells into the brain [16]. An important factor for investigating this disease is the development of suitable in vitro systems mimicking the abovementioned barriers. Whereas human models of the BBB employing immortalized cell lines have been developed [17-19], in vitro systems mimicking the BCSFB are limited to animal models, including rat cell lines and primary porcine choroid plexus epithelial cells (PCPEC) [5,20-23] (an extensive recent review covering BCSFB in vitro models is provided by Strazielle and Ghersi-Egea [24]).

The cultivation of PCPEC in medium with reduced serum concentrations improves the morphological polarity of plexus epithelial cells as well as the barrier forming cell-cell interactions. This can be clearly demonstrated by an increase in transepithelial resistance (TEER) values and a decrease in macromolecular permeability [5]. We have used this culture system for the first time to study the pathogenesis of bacterial meningitis, and ideally use S. suis as a relevant pathogen causing disease in pigs and humans. S. suis is a well characterized swine pathogen causing a wide range of infections such as meningitis, septicemia, arthritis and pneumonia [25].

Recently a human choroid plexus papilloma cell line (HIBCPP) was established [26] and has now been characterized for its suitability as BCSFB model system by our group [27]. It was demonstrated that HIBCPP, when cultured under appropriate conditions, displays several features of a functional BCSFB including the formation of TJs and the development of a high TEER concomitant with a low permeability for macromolecules when grown on cell culture inserts.
To study the pathogenesis of CNS inflammation at the porcine and even more relevant the human BCSFB, the development of an inverted culture system was necessary. By this means the investigation of the physiological interaction of pathogens or leukocytes with the basolateral cell side of the choroid plexus epithelial cells is possible. In this study protocol article we describe the experimental setup of our inverted BCSFB models and the possibilities of experimental analysis with this system.

\section{Methods}

In this section we describe our experimental setup for analysis of leukocyte transmigration in BCSFB in vitro models as summarized in Figure 1. In brief, after seeding and culturing PCPEC on the basolateral side of the culture insert membrane, cells are stimulated and barrier function and morphology analyzed for the duration of the experiment. Leukocyte transmigration is determined in the presence or absence of a chemoattractant by fluorescence quantification and imaging. Each experimental step is described in the following paragraphs in more detail.

\section{Preparation and cultivation of PCPEC on inverted cell culture inserts}

Epithelial cells from porcine choroid plexus are obtained by a modified preparation protocol [21]. Brains from freshly slaughtered pigs are dissected and the choroid plexus tissue from the lateral and the fourth ventricles removed, transported with HBSS in $\mathrm{Ca}^{2+} / \mathrm{Mg}^{2+}$ (Gibco BRL, Eggstein, Germany) and HEPES buffer (BioWhittaker, Apen) to the laboratory, and treated hereafter with mixed cold and warm trypsinisation $(0.2 \%$ solution, Biochrom, Berlin, Germany, $45 \mathrm{~min}$. at $4^{\circ} \mathrm{C}, 20 \mathrm{~min}$. at $37^{\circ} \mathrm{C}$ ). After termination of the trypsin digestion with an appropriate volume of fetal calf serum (FCS) the cells are centrifuged at $20 \times \mathrm{g}$ and resuspended in DMEM/HAM's F12 1:1 (Sigma-Aldrich, Deisenhofen, Germany) supplemented with 4 mM L-glutamine (Gibco BRL, Eggstein, Germany), 10\% heat-inactivated FCS (Biochrom KG seromed, Berlin, Germany), $5 \mu \mathrm{g} / \mathrm{ml}$ insulin (Sigma-Aldrich, Deisenhofen, Germany), and penicillin (100 U/ml)/streptomycin $(100 \mu \mathrm{g} /$ ml) (ICN Biomedicals GbbH, Eschwege, Germany). The medium is supplemented with $20 \mu \mathrm{M}$ of cytosinearabinoside (Cell Pharm GmbH, Hannover, Germany) to suppress the growth of contaminating fibroblast-like cells. The cells are seeded on laminin (BD, Le Pont De Claix, France) coated cell culture inserts (pore diameter $3.0 \mu \mathrm{m}$, $0.33 \mathrm{~cm}^{2}$; Falcon, BD, Le Pont De Claix, France) that are flipped over and placed in a medium flooded 12-well plate. For laminin-coating, $35 \mu \mathrm{l}$ of a $50 \mu \mathrm{g} / \mathrm{ml}$ solution of laminin are applied to lower filter side with subsequent drying overnight. Cells are concentrated in $100 \mu \mathrm{l}$ medium, using a 


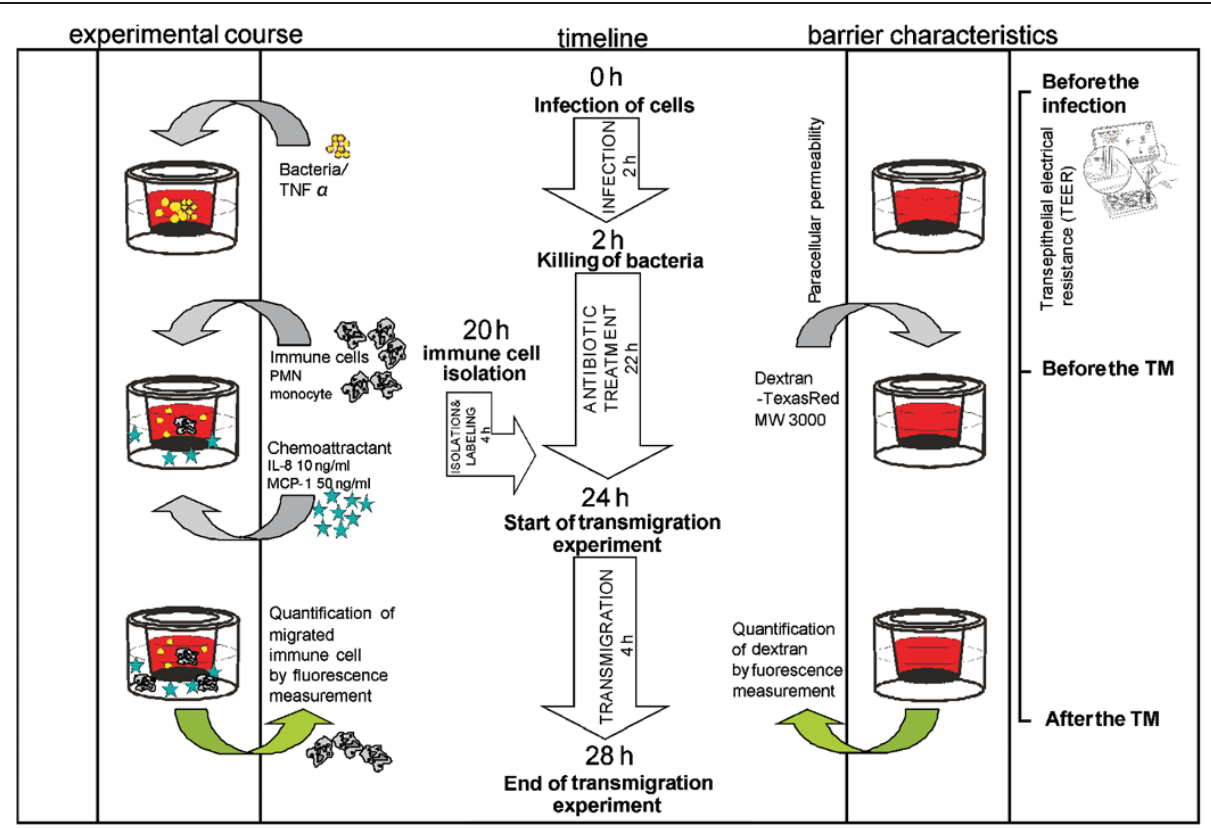

Figure 1 Experimental setup of the analysis of leukocyte transmigration in porcine and human inverted culture system with PCPEC and HIBCPP, respectively. The image of the Ohm voltohmmeter is a courtesy of EMD Millipore cooperation. Abreviations: IL-8 = Interleukin-8; $\mathrm{MCP}-1$ = monocyte chemotactic protein-1; MW = molecular weight; $\mathrm{TM}=$ transmigration.

seeding density of $60 \mathrm{~cm}^{2} / \mathrm{g}$ wet weight of choroid plexus tissue, are fed the following day and the culture insert are flipped over again on day 2 after plating. Upon confluence, PCPEC have a seeding density of approximately $1 \times 10^{5}$ cells $/ \mathrm{cm}^{2}$ (evaluated by DAPI staining of the cell nuclei using immunofluorescence imaging as described below). In the following cells are cultivated until reaching a TEER $110 \Omega \times \mathrm{cm}^{2}$, hereafter switched to medium with FCS $1 \%$, and finally used for the experiments 3-5 days later when TEER becomes greater than $200-300 \Omega \times \mathrm{cm}^{2}$.

\section{Cultivation of HIBCPP on cell culture inserts}

HIBCPP are cultured in DMEM/HAM's F12 1:1 supplemented with $4 \mathrm{mM} \mathrm{L}$-Glutamine, $5 \mu \mathrm{g} \mathrm{ml}^{-1}$ insulin, penicillin $(100 \mathrm{U} / \mathrm{ml})$ and streptomycin $(100 \mu \mathrm{g} / \mathrm{ml}), 15 \%$ heat inactivated fetal calf serum (FCS) [HIBCPP-medium with $15 \%$ FCS]. Since HIBCPP have been described to change doubling time with increasing passages [26] only cells between passage 33 and 37 are used. For culture insert-based assays the cells are seeded on cell culture inserts (pore diameter $3.0 \mu \mathrm{m}$, pore density $2.0 \times 10^{6}$ pores per $\mathrm{cm}^{2}$, membrane diameter $0.33 \mathrm{~cm}^{2}$; Greiner Bio-One, Frickenhausen, Germany). Since HIBCPP can form papillary-like structures and grow in multilayers [26], seeding of HIBCPP needing extensive trypsinization to allow formation of a maximal proportion of a monolayer on the cell culture inserts. Cells are trypsinized with trypsin $0.25 \%$ (Live technologies, Darmstadt, Germany) for $12-15$ min (up to 25 minutes) at $37^{\circ} \mathrm{C}$, washed hereafter and seeded onto filters at a seeding density of $4 \times 10^{4} /$ well or $2 \times 10^{5} /$ well depending on the time point planned for the experiment. Subsequently, cells are washed once each of the following two days. Medium is not added to the lower well before day two after seeding. For the inverted cell culture insert system the cells are basically treated as described above for PCPEC [7]. Upon confluence, HIBCPP have a seeding density of approximately $1.21 \times 10^{6}$ cells $/ \mathrm{cm}^{2}$ (evaluated by 4,6-diamidino-2-phenylindole staining of the cell nuclei using immunofluorescence imaging). When TEER values become greater than $70 \Omega \times \mathrm{cm}^{2}$, cell culture is continued in HIBCPP-medium containing $15 \%$, $1 \%$ or $0 \%$ FCS. Cells can be used for experiments 1 or 2 days later when the TEER is around $500 \Omega \times \mathrm{cm}^{2}$.

\section{Measurement of transepithelial electrical resistance (TEER)} Barrier properties of PCPEC monolayers are documented by measuring TEER. TEER can be determined using an epithelial tissue voltohmmeter $\left(\mathrm{EVOM}^{\circledR}{ }^{\circledR}\right.$, World Precision Instruments, Sarasota, FL, USA) and an STX-2 electrode system. When TEER values reach more than 200-300 $\Omega \times \mathrm{cm}^{2}$, PCPEC inverted cultures are suitable for use. In PMN transmigration experiments, TEER is monitored for $4 \mathrm{~h}$. The electrical resistance for cells in medium alone is used as the negative control and remains stable during all experiments.

\section{Determination of paracellular permeability}

Paracellular permeability across cell monolayers in the basolateral-to-apical direction is determined during 
PMN transmigration experiments using Texas Redlabelled dextran (MW 3000; Sigma, Deisenhofen, Germany). For this purpose, Texas Red-dextran (TRdextran, $100 \mu \mathrm{g} / \mathrm{ml}$ ) is loaded into the upper compartment prior to the incubation period. At different time intervals, samples from the lower compartment can be collected and fluorescence measured in a Tecan Infinite M200 Multiwell reader (Tecan, Switzerland). The percentage of dextran flux is calculated in relation to an internal standard. TEER and permeability measurements can be performed with the same cultures as used for PMN transmigration.

\section{Isolation of PMN}

For the PMN transmigration assay, blood is taken from freshly slaughtered pigs at the abattoir following the ethical guidelines. The blood is collected into sodium-heparin coated tubes (2500U/50 ml blood). Hereafter, PMN are isolated from non-coagulated citrate blood by Percoll density sedimentation according to the manufacturer's instructions (Biochrom, Berlin, Germany). Contaminating erythrocytes are lysed with $\mathrm{NH}_{4} \mathrm{Cl}$ on ice. After centrifugation $\left(10 \mathrm{~min}, 300 \mathrm{~g}, 4^{\circ} \mathrm{C}\right)$, the lysed erythrocytes are removed and the PMN pellet is washed with PBS. PMN are resuspended in culture medium at a cell density of $1 \times 10^{7} / \mathrm{ml}$. For transmigration assays, PMN are loaded with the fluorochrome 2,7'-bis-(2-carboxyethyl)-5(and-6)-carboxyfluorescein, acetomethyl ester (BCECFAM; Molecular Probes, Eugene, OR, USA) at a concentration of $1 \mu \mathrm{M}$ for $15-30 \mathrm{~min}$ at $37^{\circ} \mathrm{C}$ according to the manufacturer's instructions. After the staining period, cells are washed with culture medium and counted with a möLab cell counter (möLab, Langenfeld, Germany).

\section{Stimulation of PCPEC}

In our experimental setup, PCPEC are stimulated with either TNF $\alpha$ (R\&D Systems, USA) from the apical and basolateral side $(10 \mathrm{ng} / \mathrm{ml})$ for $24 \mathrm{~h}$ or basolaterally (blood-side) infected with $S$. suis strain 10 with an multiplicity of infection (MOI) of 10 and hereafter incubated for $2 \mathrm{~h}$ at $37^{\circ} \mathrm{C}$ and $5 \% \mathrm{CO}_{2}$. After the incubation period penicillin/streptomycin $(100 \mathrm{U} / \mathrm{ml} / 100 \mu \mathrm{g} / \mathrm{ml})$ is added to the upper and lower compartment of the cell culture insert to inhibit further extracellular bacterial growth and therefore to prevent cytotoxic effects. PMN transmigration assays are performed after an additional $22 \mathrm{~h}$ on the following day.

\section{PMN transepithelial migration assay}

For transepithelial migration assays, BCECF-AM-loaded PMN are added to the upper cell culture insert compartment (blood-side) of control, TNF $\alpha$ or S. suis stimulated cells in a PMN:PCPEC ratio of 10:1. As chemoattractant IL-8 (R\&D Systems, USA) is used at $10 \mathrm{ng} / \mathrm{ml}$ and added to the lower cell culture insert compartment (CSF-side) $30 \mathrm{~min}$ before starting the transmigration experiments. After $4 \mathrm{~h}$ of transmigration the cell culture inserts are removed and the 24-well plates are centrifuged $(5 \mathrm{~min}$, $300 \mathrm{x} \mathrm{g}$ ) to ensure that all PMN are attached to the bottom of the wells. The supernatants are collected for permeability measurements. The PMN are washed once with HBSS with $\mathrm{Ca}^{2+} / \mathrm{Mg}^{2+}$ and again centrifuged (5 min, $300 \mathrm{x} \mathrm{g}$ ). Transmigrated PMN are lysed by $1 \%$ Triton X-100 in PBS and quantified by fluorescence measurement with a Tecan $200 \mathrm{M}$ Infinite Multiwell reader (Tecan, Switzerland) in relation to an internal standard.

\section{Immunofluorescence}

Confluent PCPEC are grown on inverted cell culture inserts, stimulated with S. suis or TNF $\alpha$ and co-cultured with PMN as described above. After $4 \mathrm{~h}$ of transmigration towards a gradient of IL-8, the cells are washed, fixed with formaldehyde $4 \%$ (10 $\mathrm{min})$ and hereafter washed again. The filters can principally be stored at $4{ }^{\circ} \mathrm{C}$ until final staining. Filter membranes are cut out of the filters and cells are permeabilized with $0.5 \%$ Triton X$100 / 1 \%$ BSA in PBS at room temperature for $1 \mathrm{~h}$. Subsequently, the filters are washed again with PBS without $\mathrm{Ca}$ and $\mathrm{Mg}$ and incubated overnight at $4^{\circ} \mathrm{C}$ with the primary antibodies to stain the TJ proteins. Occludin or ZO-1 rabbit antibodies $(1 \mu \mathrm{g} / \mathrm{ml})$ are used at a dilution of 1:250 (Zymed Laboratories, San Francisco, USA). On the following day the cells are washed again, incubated for $60 \mathrm{~min}$ with the secondary antibody 1:1000 (Alexa fluor $^{\circledR} 594$ goat anti-chicken; Molecular probes, Karlsruhe, Germany), with Phalloidin Alexa fluor ${ }^{\circledR} 660$ (Invitrogen, Paisley, UK) for staining the actin cytoskeleton and with 4-6-diamidino-2-phenylindole dihydrochloride (DAPI) (Calbiochem, Merck KGaA, Darmstadt, Germany) (1:25.000) for staining nuclei. PMN are labelled with the granulocyte-monocyte marker SWC3a-FITC (1:100 in PBS) (Southern Biotech, Birmingham, AL, USA) for $30 \mathrm{~min}$. After washing the cells three times with PBS the culture inserts are embedded in ProLongAntifadeReagent (Invitrogen, Karlsruhe, Germany). Images are acquired with Zeiss Apotome ${ }^{\circledR}$ and Axiovision software (Carl Zeiss, Jena, Germany) using a 63x/1.4 NA objective lens. The image acquisition is carried out using the Zeiss scanning software Axiovison 4.6 and Axiovison module Inside 4D.

\section{Scanning electron microscopy}

Samples are fixed with $2.5 \%$ glutaraldehyde in cacodylate buffer, postfixed with $1 \%$ osmium tetroxide in phosphatebuffered saline, dehydrated in a graded series of ethanol and critical-point-dried with $\mathrm{CO}_{2}$. Finally the samples are sputter-coated with a layer of $7 \mathrm{~nm}$ gold/palladium 
(Bal-Tec MED 010) and examined at $20 \mathrm{kV}$ accelerating voltage in a Hitachi S-800 field emission scanning electron microscope.

\section{Troubleshooting}

Several factors have to be taken into consideration, if implausible results in the transmigration experiments with PCPEC or HIBCPP are observed. Preferentially, freshly-isolated PMN should be used to guarantee a high cell viablility. In case of a high red blood cell contamination, a second step of erythrocyte lysis should also be performed. However, the lysis should be not perfomed for too long, since it may otherwise also endanger cell viablility. A TEER between $200-300 \Omega \mathrm{x} \mathrm{cm}^{2}$ in the case PCPEC and a TEER between 200-500 $\Omega \mathrm{x} \mathrm{cm}^{2}$ in the case of HIBCPP are optimal for performing the transmigration experiments. Lower or high values may deliver sub-optimal results. Daily monitoring of TEER guarantees determination of the time-point most suited for the start of experiment. Before a bacterial infection experiment is initiated, the filters have to be thoroughly washed with antibiotic-free medium.

\section{Discussion}

One crucial step in the pathogenesis of inflammatory diseases of the CNS is the excessive infiltration of leukocytes into the CSF bearing severe consequences. To study the interaction of pathogens or leukocytes with the basolateral cell side of the choroid plexus epithelial cells in vitro, the development of an inverted cell culture system became necessary.

Only a few inverted culture insert systems exist so far that enable investigation of leukocyte transmigration across cellular barriers. Most of the models use epithelial cell lines although primary cells are occasionally used also [28]. In general, leukocytes isolated from peripheral blood can be added to the upper chamber of the culture insert and stimulated to migrate toward a chemoattractant [e.g., n-formyl-methionyl-leucyl-pheny- lalanine (fMLP), interleukin (IL)-8, C5a, leukotriene B4, IL1 $\beta$, MCP-1, CXCL-12] applied to the lower reservoir. Depending on which side of the filter the cells are cultured, transmigration can be modelled in either the apical-tobasolateral or the basolateral-to-apical direction. The surface area and filter pore sizes can be varied, but typically filters with a surface area of $0.33 \mathrm{~cm}^{2}$ and pore sizes of 3.0-5.0 $\mu \mathrm{m}$-large enough to allow the passage of leukocytes-are used. Culture inserts with pore sizes that are too small to allow leukocyte transepithelial migration to occur may be used to determine the effects of soluble factors or intercellular contact on cellular functions [29-32].

Recently, we established an inverted cell culture insert model of PCPEC and HIBCPP that display robust barrier properties and enable investigation of pathogen and leukocyte transmigration in the physiologically- relevant basolateral-to-apical direction $[7,27]$. Of note, we also established a novel porcine choroid plexus epithelial cell line (PCP-R), which displays important features of a functional BCSFB in vitro model, i.e. the formation of a strong barrier function as demonstrated by a high TEER and a low permeability for macromolecules [33]. In preliminary experiments, this cell line seems also to be suitable for the establishment of an inverted cell culture system.

As a model of an inflammatory event in the CNS, we established bacterial infection with the zoonotic pathogen S. suis mimicking bacterial meningitis in our porcine model. Here, we could demonstrate that these bacteria specifically invade PCPEC from the basolateral side in a capsule-dependent manner [7]. Inflammatory activation of epithelial and endothelial cells, e.g. after bacterial infection, induces the release of interleukin-8 (IL-8/ CXCL8) and other chemokines that recruit polymorphonuclear neutrophils (PMN) $[28,34]$. The chemokine IL-8 is known to interact with its cognate receptors CXCR1 and CXCR2. CXCR2 is the main receptor involved in neutrophil chemotaxis, leading to cell migration into the brain during injury, infection or disease [35]. Therefore IL-8 seemed to be a suitable chemoattractant to study PMN transmigration at the BCSFB in our model in vitro. In fact, IL-8 induced a strong chemotaxis in our transmigration experiments [36].

Employing immunofluorescence and electron microscopic studies we could now show for the first time in an in vitro model of the BCSFB that PMN can traverse epithelial monolayers transcellularly. An example for analysis by immunofluorescence is shown in Figure 2, demonstrating that PMN are either localized within the cell body of stimulated PCPEC, surrounded by tight junctions and actin, or are found close to tight junctions. Rarely, tight junction disarrangement is demonstrated in areas of PMN transversal. Views from above on the apical cell side in 3D images show in detail transcellular PMN migration, in which the centrally localized PMN migrates distal to the TJ. The sideview indicates that the PMN is about to leave the cell (Figure 3). Extensive sequential analyses of the PMN transmigration process with Apotome ${ }^{\circledR}$-imaging and electron microscopy revealed that paracellular migrating PMN stop just before tight junctions. Interestingly, PMN subsequently appeared to proceed by transcellular migration via funnel-like structures developing from the apical membrane [36]. Moreover, in scanning electron microscopic analysis we frequently observed PMN in clear distance to intercellular borders (Figure 4). Unlike for transendothelial migration, there was until recently no evidence that PMN take the transcellular route through epithelial cells. For 


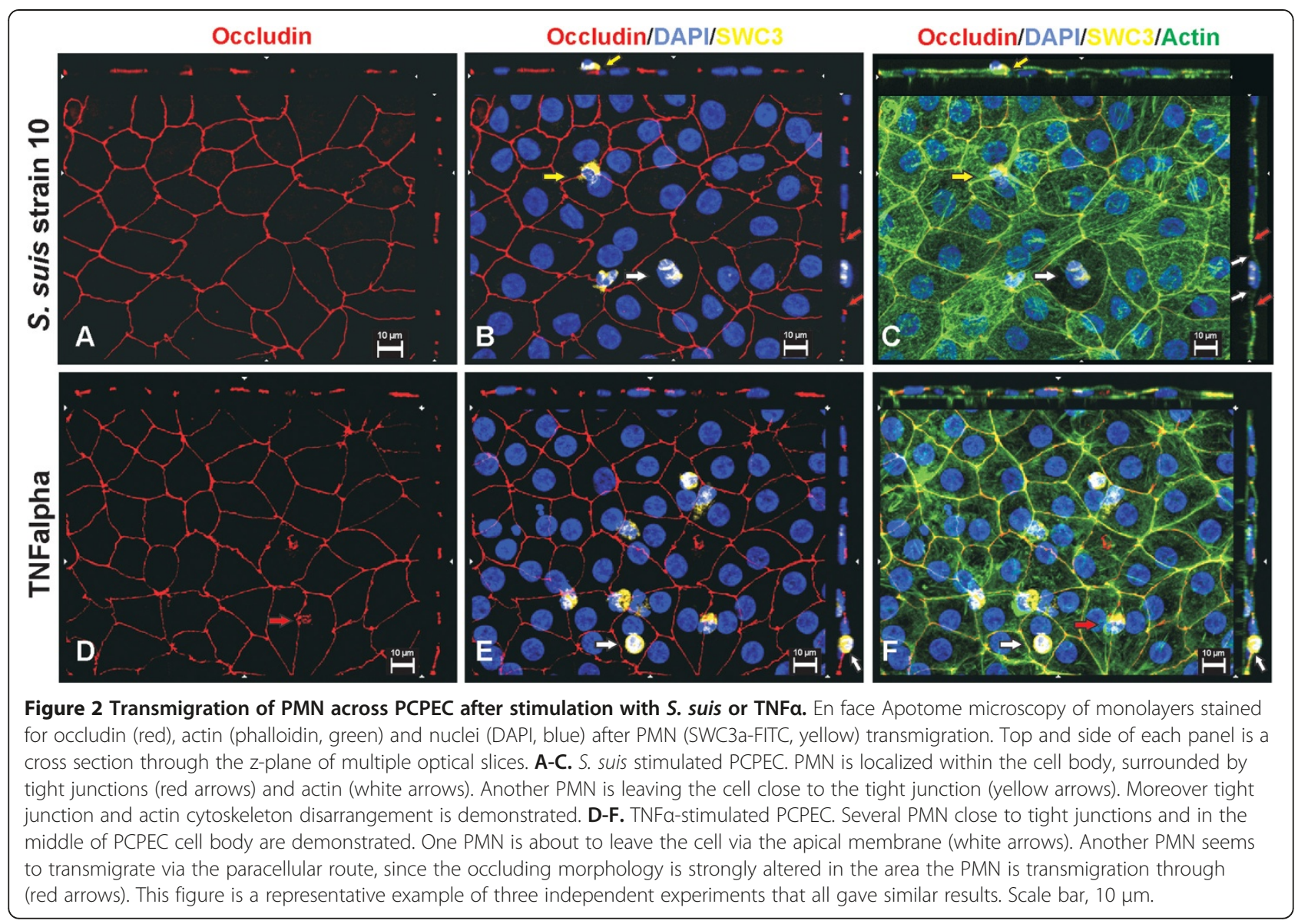

transendothelial migration the paracellular route between adjacent cells was postulated for a long time, but in the meanwhile the transcellular route directly through the endothelial cell body has been well documented $[37,38]$. The exact mechanisms for the transcellular migration of PMN through PCPEC, especially the roles of adhesion and tight junction molecules, remain to be elucidated further.

Of note, in the human BCSFB model, a polar invasion of $N$. meningitidis from the physiologically-relevant basolateral cell side could also be demonstrated [27]. Currently, experiments are in progess in the human inverted cell culture system to analyze the mechanisms of PMN and monocyte transmigration across HIBCPP after infection with $N$. meningitidis. In the same model, we recently analysed the permissiveness of plexus epithelial cells for Echovirus 30 (EV30). Analysis of the cytokine/chemokine-profile following enteroviral infection with a cytometric bead array and Q-PCR revealed an enhanced secretion of PanGRO (CXCL1, CXCL2 and CXCL3), IL8 and CCL5. However, there was only a minor effect of HIBCPP-infection with EV30 on transepithelial $\mathrm{T}$ lymphocyte migration with or without the chemoattractant CXCL12. Moreover, CXCL3 did not significantly enhance $\mathrm{T}$ cell migrations [39].

In the following we describe and discuss the differences between the known inverted culture transwell models that study leukocyte transmigration in vitro. Epithelial monolayers such as intestinal epithelial T84 cells have been shown to grow in upright or inverted fashion on collagen-coated permeable supports. In order to grow the T84 on the filter bottom the culture insert cup was inverted and fitted with a tight plastic collar then placed in a bath of medium, which was added also into the collar. T84 cells were harvested and cells were added to the collagen-coated surface of each culture insert which was then left to further incubate. The collars were subsequently removed and the culture inserts righted and placed into a 24-well plate with fresh media [29-31]. Since plastic collars seem easy to handle this tool has frequently been used.

In a lung in vitro model, human airway mucoepidermal cell line H292 or human primary bronchial epithelial cells (HBEC) were also cultured as inverted monolayers [40], according to Parkos and colleagues [29], with minor modifications [41]. Apical stimulation 


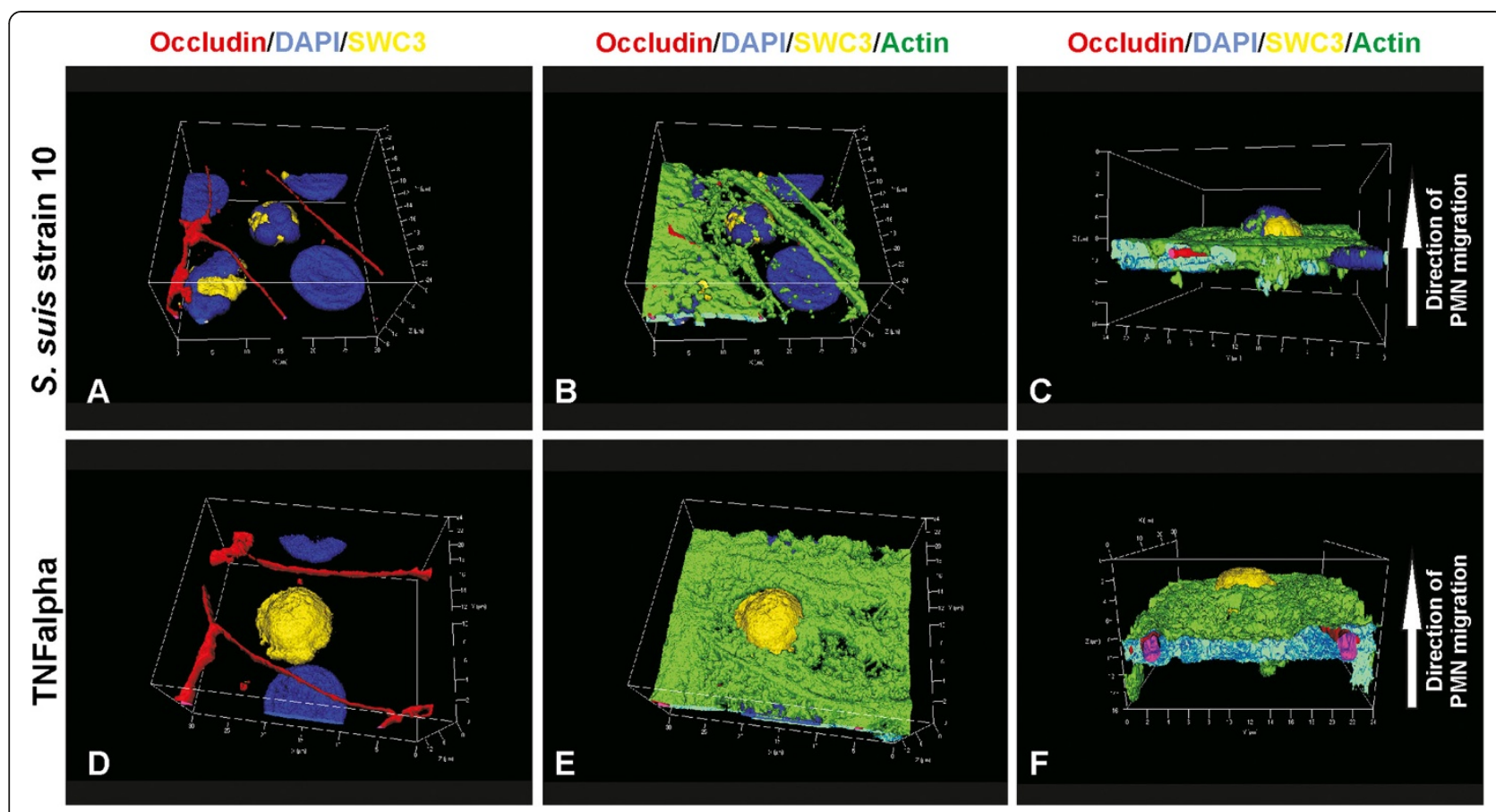

Figure 3 3-D Analysis of PMN transmigration across PCPEC. Three-dimensional immunofluorescence images of stimulated PCPEC co-cultured with PMN were reconstructed from $0.3 \mu \mathrm{m}$ Apotome optical sections, using Zeiss software Inside 4D. A-C. S. suis stimulated PCPEC. Views from above on the apical cell side $(\mathbf{A}, \mathbf{B})$ show transcellular PMN migration, in which the centrally localized PMN migrates distal to the TJ. The sideview indicated that the PMN is about to leave the cell (C). D-E. TNFa-stimulated PCPEC. A centrally localized PMN (A,B), that transmigrates from the basolateral to the apical direction $(\mathbf{C})$ is demonstrated. This figure is a representative example of three independent experiments that all gave similar results. Scale bar, as indicated.

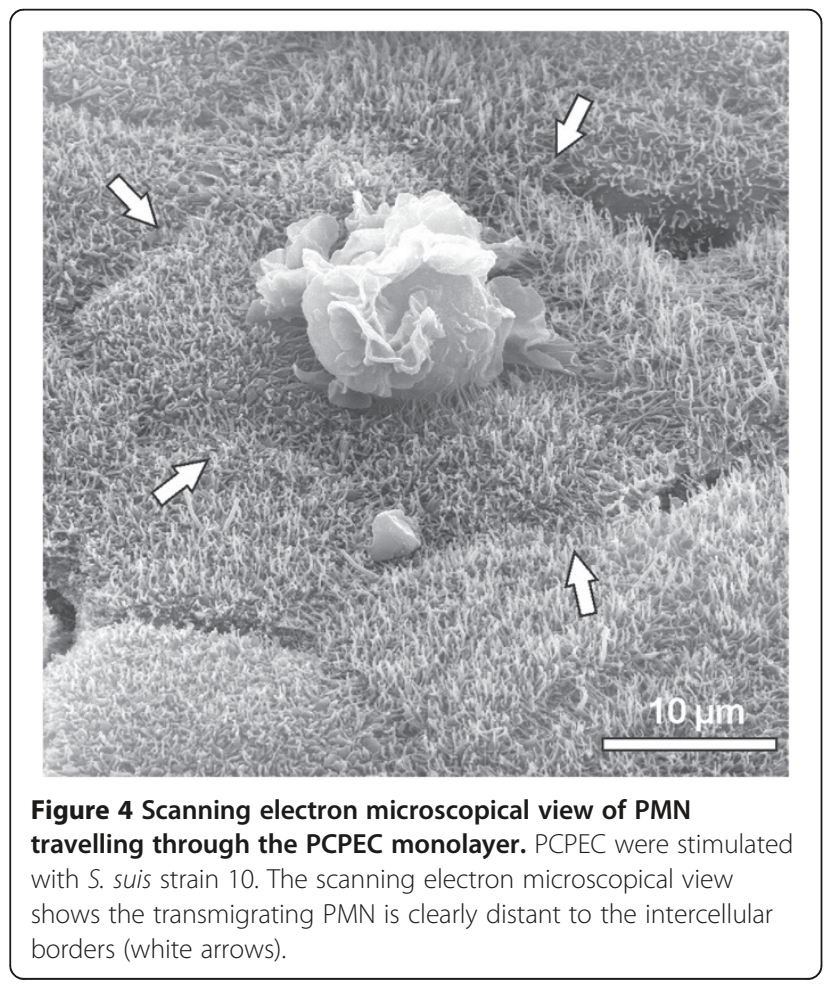

of the monolayer was performed with IL-1ß and PMN were labelled with ${ }^{51} \mathrm{Cr}$. The fluids from both chambers as well as that of the culture inserts were collected separately after the transmigration experiment and hereafter the amount of ${ }^{51} \mathrm{Cr}$ was determined in a gamma counter, which has the advantage of precise analysis, but also disadvantage of the requirement for radioactive facilities [40]. Calu-3 cells as well as primary human ATII cells were also previously used in inverted culture insert systems [34]. Here, the chemoattractant fMLP was added to the lower chamber. After migration, epithelial cells were purified from PMN by magnetic-activated cell sorting separation using anti-epithelial cell adhesion molecule antibodies. This form of analysis requires special equipment and may not be applicable for broad use. Another lung infection model consisting of alveolar epithelial cells (A549) and human PMN grown on inverted culture inserts was used to determine whether also bacteria such as Pseudomonas aeroginosa, Klebsiella pneumonia and E. coli are capable of inducing PMN migration across these epithelial barriers [42]. PMN that fully migrated into the apical chamber were also quantified by the myeloperoxidase assay as used by others $[32,43]$.

To optimize the lung in vitro model, Mul et al. developed a bilayer transmigration model composed of 
primary human endothelial (human papilloma virusimmortalized HUVEC cell line or freshly isolated, primary HUVECs) and lung epithelial cells (H292 or primary bronchial epithelial cells), simultaneously cultured on opposite sides of culture inserts [44]. PMN were labelled with calcein-AM prior to the start of the transmigration assay and after lysis the amount of fluorescence in each of culture insert compartments or attached cells was measured in a spectrofluorimeter at the end of the experiment. All in all, bilayer models are more in vivo like, than single cell models, and therefore may be preferred. However, from our experience, establishment of such a system is demanding, since culture conditions may differ significantly among the cells and may lead to interference.

In contrast to the described inverted systems above PCPEC were cultured on laminin coated culture inserts and no plastic collar was used. PMN were labelled with BCECF-AM that can easily determined by fluorescence analysis. Since measurement of fluorescence is easy to perform, labelling of PMN with a fluorescent marker has been proven to be a very feasible option. Additionally, labelled PMN can afterwards also be analysed in immunofluorescence experiments in combination with other stainings (e.g. occludin). Another advantage of this experimental setup is that barrier function can be monitored in parallel. In future experiments a similar experimental protocol can be used in the human BCSFB system with cultured HIBCPP.

In conclusion, our inverted cell culture system of the choroid plexus epithelial cells enables investigation of pathogen as well as leukocyte interaction at the BCSFB barrier. The methodology seems applicable and has useful modifications of already-existing systems. Recent functional and morphological analysis of PMN transmigration after bacterial stimulation as well as T-cell transmigration after viral infection support the value of this system. Especially the human model offers now a wide range of options for analysis of CNS disease-related mechanisms at the choroid plexus epithelium.

\footnotetext{
Abbreviations

BBB: Blood-brain barrier; BCECF-AM: 2',7'-bis-(2-carboxyethyl)-5-(and-6)carboxyfluorescein, acetomethyl ester; BCSFB: Blood-cerebrospinal fluid barrier; CNS: Central nervous system; CSF: Blood-cerebrospinal fluid; DAPI: 4'6-diamidino-2-phenylindole dihydrochloride; EAE: Experimental autoimmune encephalomyelitis; HIBCPP: Human choroid plexus papilloma cells; IL8: Interleukin-8; MCP-1: Monocyte chemotactic protein-1; MS: Multiple sclerosis; MW: Molecular weight; PCPEC: Primary porcine choroid plexus epithelial cells; PMN: Polymorphonuclear neutrophils; TEER: Transepithelial electrical resistance; TJs: Tight junctions; TM: Transmigration.
}

\section{Competing interests}

The authors declare that they have no competing interests.

\section{Authors' contributions}

TT conceived and coordinated the study, and drafted the manuscript. CW US cell culture and immunofluorescence experiments. JB and US performed the electron microscopic studies. CS and HS have co-conceived the study and have been involved in drafting the manuscript. All authors have read and approved the final version of this manuscript.

\section{Acknowledgements}

This work was supported by grants from the Deutsche Forschungsgemeinschaft (TE 653/2-2). The authors wish to thank Prof. Hartwig Wolburg for the support, and the technicians A. Seibt for expert advice.

\section{Author details}

'Paediatric Infectious Diseases, Department of Pediatric and Adolescent Medicine, Universitätsmedizin Mannheim, Medical Faculty Mannheim, Heidelberg University, Theodor-Kutzer-Ufer 1-3, Mannheim, Germany. ${ }^{2}$ Institut für Genetik von Herzerkrankungen (IfGH), Department für Kardiologie und Angiologie, Universitätsklinikum Münster, Münster, Germany. ${ }^{3}$ Max Planck Institute of Developmental Biology, Tuebingen, Germany.

Received: 30 August 2012 Accepted: 12 November 2012

Published: 10 January 2013

\section{References}

1. Balda MS, Matter K: Tight junctions. J Cell Sci 1998, 111(Pt 5):541-547.

2. Strazielle N, Ghersi-Egea JF: Choroid plexus in the central nervous system: biology and physiopathology. I Neuropathol Exp Neurol 2000, 59(7):561-574.

3. Engelhardt B, Wolburg-Buchholz K, Wolburg H: Involvement of the choroid plexus in central nervous system inflammation. Microsc Res Tech 2001, 52(1):112-129.

4. Abbott NJ, Patabendige AA, Dolman DE, Yusof SR, Begley DJ: Structure and function of the blood-brain barrier. Neurobiol Dis 2010, 37(1):13-25.

5. Haselbach M, Wegener J, Decker S, Engelbertz C, Galla HJ: Porcine Choroid plexus epithelial cells in culture: regulation of barrier properties and transport processes. Microsc Res Tech 2001, 52(1):137-152.

6. Tenenbaum T, Matalon D, Adam R, Seibt A, Wewer C, Schwerk C, Galla HJ, Schroten H: Dexamethasone prevents alteration of tight junctionassociated proteins and barrier function in porcine choroid plexus epithelial cells after infection with Streptococcus suis in vitro. Brain Res 2008, 1229:1-17.

7. Tenenbaum T, Papandreou T, Gellrich D, Friedrichs U, Seibt A, Adam R, Wewer C, Galla HJ, Schwerk C, Schroten H: Polar bacterial invasion and translocation of Streptococcus suis across the blood-cerebrospinal fluid barrier in vitro. Cell Microbiol 2009, 11(2):323-336.

8. Pron B, Taha MK, Rambaud C, Fournet JC, Pattey N, Monnet JP, Musilek M, Beretti IL, Nassif $X$ : Interaction of Neisseria maningitidis with the components of the blood-brain barrier correlates with an increased expression of PilC. J Infect Dis 1997, 176(5):1285-1292.

9. Smith AL: Pathogenesis of Haemophilus influenzae meningitis. Pediatr Infect Dis J 1987, 6(8):783-786.

10. Parkkinen J, Korhonen TK, Pere A, Hacker J, Soinila S: Binding sites in the rat brain for Escherichia coli $S$ fimbriae associated with neonatal meningitis. J Clin Invest 1988, 81(3):860-865.

11. Prats N, Briones V, Blanco MM, Altimira J, Ramos JA, Dominguez L, Marco A: Choroiditis and meningitis in experimental murine infection with Listeria monocytogenes. Eur J Clin Microbiol Infect Dis 1992, 11(8):744-747.

12. Sanford SE: Gross and histopathological findings in unusual lesions caused by Streptococcus suis in pigs. II. Central nervous system lesions. Can J Vet Res 1987, 51(4):486-489.

13. Tabor-Godwin JM, Ruller CM, Bagalso N, An N, Pagarigan RR, Harkins S, Gilbert PE, Kiosses WB, Gude NA, Cornell CT, et al: A novel population of myeloid cells responding to coxsackievirus infection assists in the dissemination of virus within the neonatal CNS. J Neurosci 2010, 30 (25):8676-8691.

14. Zhang JR, Tuomanen E: Molecular and cellular mechanisms for microbial entry into the CNS. J Neurovirol 1999, 5(6):591-603.

15. Kim KS: Pathogenesis of bacterial meningitis: from bacteraemia to neuronal injury. Nat Rev Neurosci 2003, 4(5):376-385.

16. Wolburg H, Paulus W: Choroid plexus: biology and pathology. Acta Neuropathol 2010, 119(1):75-88. 
17. Stins MF, Badger J, Sik Kim K: Bacterial invasion and transcytosis in transfected human brain microvascular endothelial cells. Microb Pathog 2001, 30(1):19-28.

18. Weksler BB, Subileau EA, Perriere N, Charneau P, Holloway K, Leveque M, Tricoire-Leignel H, Nicotra A, Bourdoulous S, Turowski P, et al: Blood-brain barrier-specific properties of a human adult brain endothelial cell line. FASEB J 2005, 19(13):1872-1874.

19. Muruganandam A, Herx LM, Monette R, Durkin JP, Stanimirovic DB: Development of immortalized human cerebromicrovascular endothelial cell line as an in vitro model of the human blood-brain barrier. FASEB J 1997, 11(13):1187-1197.

20. Shi LZ, Zheng W: Establishment of an in vitro brain barrier epithelial transport system for pharmacological and toxicological study. Brain Res 2005, 1057(1-2):37-48.

21. Gath U, Hakvoort A, Wegener J, Decker S, Galla HJ: Porcine choroid plexus cells in culture: expression of polarized phenotype, maintenance of barrier properties and apical secretion of CSF-components. Eur J Cell Biol 1997, 74(1):68-78.

22. Kitazawa T, Hosoya K, Watanabe M, Takashima T, Ohtsuki S, Takanaga H, Ueda M, Yanai N, Obinata M, Terasaki T: Characterization of the amino acid transport of new immortalized choroid plexus epithelial cell lines: a novel in vitro system for investigating transport functions at the bloodcerebrospinal fluid barrier. Pharm Res 2001, 18(1):16-22.

23. Zheng W, Zhao Q: Establishment and characterization of an immortalized Z310 choroidal epithelial cell line from murine choroid plexus. Brain Res 2002, 958(2):371-380.

24. Strazielle N, Ghersi-Egea JF: In vitro models of the blood-cerebrospinal fluid barrier and their use in neurotoxicological research. NeuroMethods 2011, 56:161-184.

25. Staats JJ, Plattner BL, Stewart GC, Changappa MM: Presence of the Streptococcus suis suilysin gene and expression of MRP and EF correlates with high virulence in Streptococcus suis type 2 isolates. Vet Microbiol 1999, 70(3-4):201-211.

26. Ishiwata I, Ishiwata C, Ishiwata E, Sato Y, Kiguchi K, Tachibana T, Hashimoto $H$, Ishikawa $H$ : Establishment and characterization of a human malignant choroids plexus papilloma cell line (HIBCPP). Hum Cell 2005, 18(1):67-72.

27. Schwerk C, Papandreou T, Schuhmann D, Nickol L, Borkowski J, Steinmann U, Quednau N, Stump C, Weiss C, Berger J, et al: Polar invasion and translocation of Neisseria meningitidis and Streptococcus suis in a novel human model of the blood-cerebrospinal fluid barrier. PLoS One 2012, 7(1):e30069.

28. Chin AC, Parkos CA: Pathobiology of neutrophil transepithelial migration: implications in mediating epithelial injury. Annu Rev Pathol 2007, 2:111-143.

29. Parkos CA, Delp C, Arnaout MA, Madara JL: Neutrophil migration across a cultured intestinal epithelium. Dependence on a CD11b/CD18-mediated event and enhanced efficiency in physiological direction. $J$ Clin Invest 1991, 88(5):1605-1612.

30. Parkos CA, Colgan SP, Delp C, Arnaout MA, Madara JL: Neutrophil migration across a cultured epithelial monolayer elicits a biphasic resistance response representing sequential effects on transcellular and paracellular pathways. J Cell Biol 1992, 117(4):757-764.

31. Madara JL, Parkos C, Colgan S, Nusrat A, Atisook K, Kaoutzani P: The movement of solutes and cells across tight junctions. Ann N Y Acad Sci 1992, 664:47-60.

32. Blake KM, Carrigan SO, Issekutz AC, Stadnyk AW: Neutrophils migrate across intestinal epithelium using beta2 integrin (CD11b/CD18)independent mechanisms. Clin Exp Immunol 2004, 136(2):262-268.

33. Schroten M, Hanisch FG, Quednau N, Stump C, Riebe R, Lenk M, Wolburg H, Tenenbaum T, Schwerk C: A novel porcine in vitro model of the bloodcerebrospinal fluid barrier with strong barrier function. PLoS One 2012, 7(6):e39835.

34. Zemans RL, Colgan SP, Downey GP: Transepithelial migration of neutrophils: mechanisms and implications for acute lung injury. Am J Respir Cell Mol Biol 2009, 40(5):519-535.

35. Wong D, Prameya R, Dorovini-Zis K: Adhesion and migration of polymorphonuclear leukocytes across human brain microvessel endothelial cells are differentially regulated by endothelial cell adhesion molecules and modulate monolayer permeability. J Neuroimmunol 2007, 184(1-2):136-148.
36. Wewer C, Seibt A, Wolburg H, Greune L, Schmidt MA, Berger J, Galla HJ, Quitsch U, Schwerk C, Schroten H, et al: Transcellular migration of neutrophil granulocytes through the blood-cerebrospinal fluid barrier after infection with Streptococcus suis. J Neuroinflammation 2011, 8(1):51

37. Ransohoff RM, Kivisakk P, Kidd G: Three or more routes for leukocyte migration into the central nervous system. Nat Rev Immunol 2003, 3(7):569-581.

38. Engelhardt $\mathrm{B}$, Wolburg $\mathrm{H}$ : Mini-review: transendothelial migration of leukocytes: through the front door or around the side of the house? Eur J Immunol 2004, 34(11):2955-2963.

39. Schneider HWC, Schoeller J, Steinmann U, Borkowski J, Ishikawa H, Findeisen P, Adams O, Doerries R, Schwerk C, Schroten H, Tenenbaum T: Chemotaxis of T-cells after infection of human choroid plexus papilloma cells with echovirus 30 in an in vitro model of the blood-cerebrospinal fluid barrier. Virus Res 2012, 170(1-2):66-74.

40. Liu L, Mul FP, Kuijpers TW, Lutter R, Roos D, Knol EF: Neutrophil transmigration across monolayers of endothelial cells and airway epithelial cells is regulated by different mechanisms. Ann N Y Acad SCl 1996, 796:21-29.

41. Zeillemaker AM, Mul FP, van Papendrecht AA H, Leguit P, Verbrugh HA, Roos D: Neutrophil adherence to and migration across monolayers of human peritoneal mesothelial cells. The role of mesothelium in the influx of neutrophils during peritonitis. J Lab Clin Med 1996, 127(3):279-286.

42. Hurley BP, Siccardi D, Mrsny RJ, McCormick BA: Polymorphonuclear cell transmigration induced by Pseudomonas aeruginosa requires the eicosanoid hepoxilin A3. J Immunol 2004, 173(9):5712-5720.

43. McCormick BA, Hofman PM, Kim J, Carnes DK, Miller SI, Madara JL: Surface attachment of Salmonella typhimurium to intestinal epithelia imprints the subepithelial matrix with gradients chemotactic for neutrophils. J Cell Biol 1995, 131(6 Pt 1):1599-1608.

44. Mul FP, Zuurbier AE, Janssen H, Calafat J, van Wetering S, Hiemstra PS, Roos D, Hordijk PL: Sequential migration of neutrophils across monolayers of endothelial and epithelial cells. J Leukoc Biol 2000, 68(4):529-537.

doi:10.1186/2045-8118-10-1

Cite this article as: Tenenbaum et al.: Culture models to study leukocyte trafficking across the choroid plexus. Fluids and Barriers of the CNS 2013 10:1.

\section{Submit your next manuscript to BioMed Central and take full advantage of:}

- Convenient online submission

- Thorough peer review

- No space constraints or color figure charges

- Immediate publication on acceptance

- Inclusion in PubMed, CAS, Scopus and Google Scholar

- Research which is freely available for redistribution 